\title{
Correcting the PL Relation?
}

\author{
C.D. Laney \\ South African Astronomical Observatory, PO Box 9, Observatory, 7935, \\ South Africa
}

Abstract. Metal-poor Cepheids do not appear to have smaller radii or cooler temperatures than more metal-rich Cepheids. A correction to the LMC modulus of $0.4 \mathrm{mag}$, due to metal-poor Cepheids being fainter for their periods, is inconsistent with the data at the $6 \sigma$ level or greater.

Do we need large metallicity corrections to moduli determined using Cepheid $P L$ relations?

A large part of the answer hinges on the correctness of conventional theoretical predictions (as in Iben \& Renzini (1984), for example) that the bolometric $\mathrm{PL}$ relation is essentially invariant with metallicity. It has not been easy to find a practical way of testing this. The usual samples of LMC and SMC Cepheids show little evidence of any great spread in composition (Caldwell \& Coulson 1985), and the samples in M31 (Freedman \& Madore 1990) have been shown by Feast (1991) to yield rather inconclusive results. There were also few sufficiently precise metallicity measurements of Cepheids in our own Galaxy, let alone in the Clouds or beyond. Very recently, Fry \& Carney (1997) have determined highprecision metal abundances for 23 Galactic Cepheids. 16 of these have SAAO $J H K$ photometry and adequate radial velocity curves.

If Cepheids are as much as 0.4 mag fainter at the LMC metallicity, as suggested by (M.)Sekiguchi \& Fukugita (1998), then metal-poor stars must either be much smaller or much cooler or both.

To test the effect of metallicity on radius, we need accurate radii, determined on a consistent system. Laney \& Stobie (1995a,b) (LS95a,b) determined Baade-Wesselink radii for 49 galactic Cepheids using $B V R I$ and $J H K$ photometry, and showed that the radii calculated using $K$-band photometry appeared to be reasonably free of systematic errors. Since that time $J H K$ data have been obtained for additional, mostly short-period Cepheids, and radii have been calculated for about half of these new objects, while improved radii have been calculated for QZ Nor, EV Sct, SZ Tau and CV Mon. For a few stars of exceptionally low amplitude, only the $(K, V-K)$ solution has been used here. Otherwise the $(K, V-K)$ and $(K, J-K)$ solutions have been averaged where both are available.

A combined Galaxy/LMC/SMC period-radius relation for fundamental pulsators (Fig. 1) gives (with 60 Galactic and 9 MC Cepheids): 


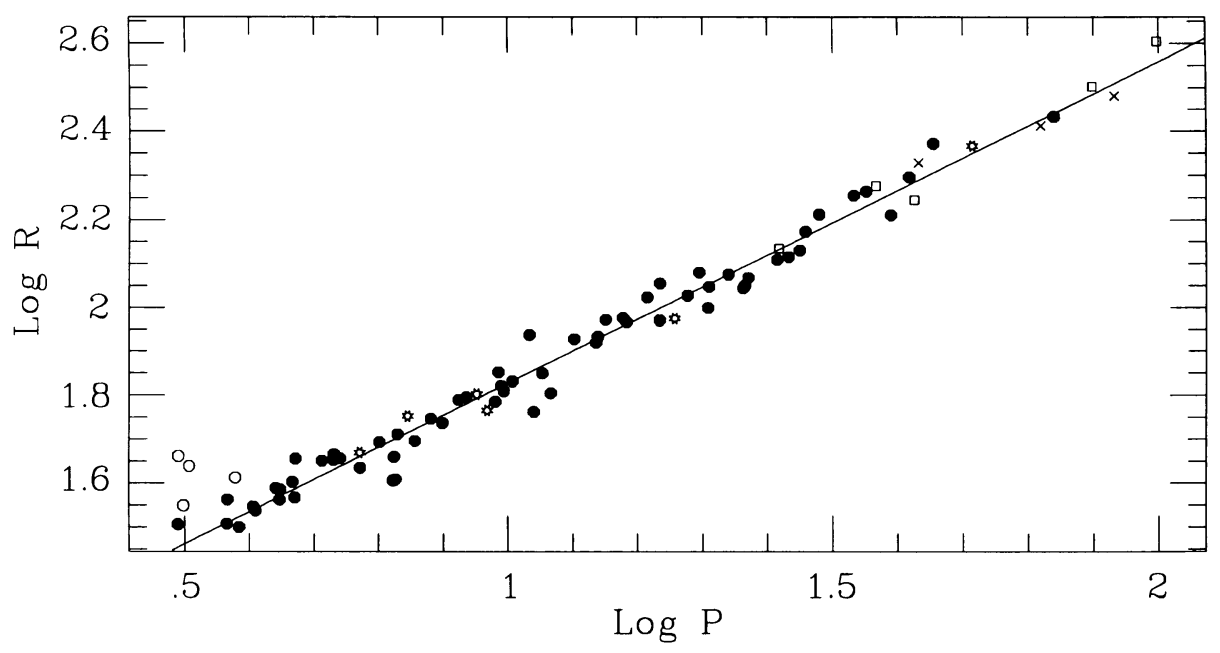

Figure 1. The Cepheid period-radius relation (solid line) for Galactic Cepheids (filled circles), LMC Cepheids (squares) and SMC Cepheids (crosses). Galactic Cepheids with less well-determined radii (asterisks) and overtone Cepheids (open circles) are also shown, but were not used in determining the P-R relation.

$$
\begin{aligned}
& \log R=1.827+0.727(\log P-1)+0.017(L M C)+0.002(S M C) \quad \sigma=0.038 . \\
& \pm 0.005 \pm 0.015 \quad \pm 0.020 \quad \pm 0.022
\end{aligned}
$$

Likely overtone pulsators and stars with relatively uncertain radii (see LS95a) have been excluded in this solution. Note that systematic differences between galactic and $\mathrm{MC}$ radii are less than $1 \sigma$, in a direction implying that $\mathrm{MC}$ Cepheids are brighter than galactic Cepheids by a few hundredths of a magnitude.

Taking our 16 Cepheids with Fry \& Carney metallicities, I now apply the above zero point and slope and find that:

$$
\begin{aligned}
& \Delta \log R=-0.007-0.107 \text { (overtones) }+0.110[\mathrm{Fe} / \mathrm{H}] \quad \sigma=0.039 \text {, } \\
& \pm 0.011 \pm 0.026 \quad \pm 0.086
\end{aligned}
$$

where $\Delta \log \mathrm{R}$ is the $\log$ of the predicted minus the $\log$ of the empirical radius (Fig. 2). Without the 3 overtone Cepheids, the coefficient of $[\mathrm{Fe} / \mathrm{H}]$ becomes $-0.05 \pm 0.11$. For an $\mathrm{LMC}[\mathrm{Fe} / \mathrm{H}]$ of -0.2 , the log of the radius will be larger by $0.02 \pm 0.02$ (magnitude brighter by 0.11 ) in the first case, smaller by $0.01 \pm 0.02$ (magnitude fainter by 0.05 ) in the second. The latter result is exactly what would be expected if the bolometric PL relation were precisely invariant, since the equations of Iben \& Renzini imply a slightly higher temperature (and surface brightness) at a given period for Cepheids of lower metallicity. If we include all 16 stars, the marginal $1 \sigma$ trend would again make low-metallicity Cepheids slightly bright for their periods. 


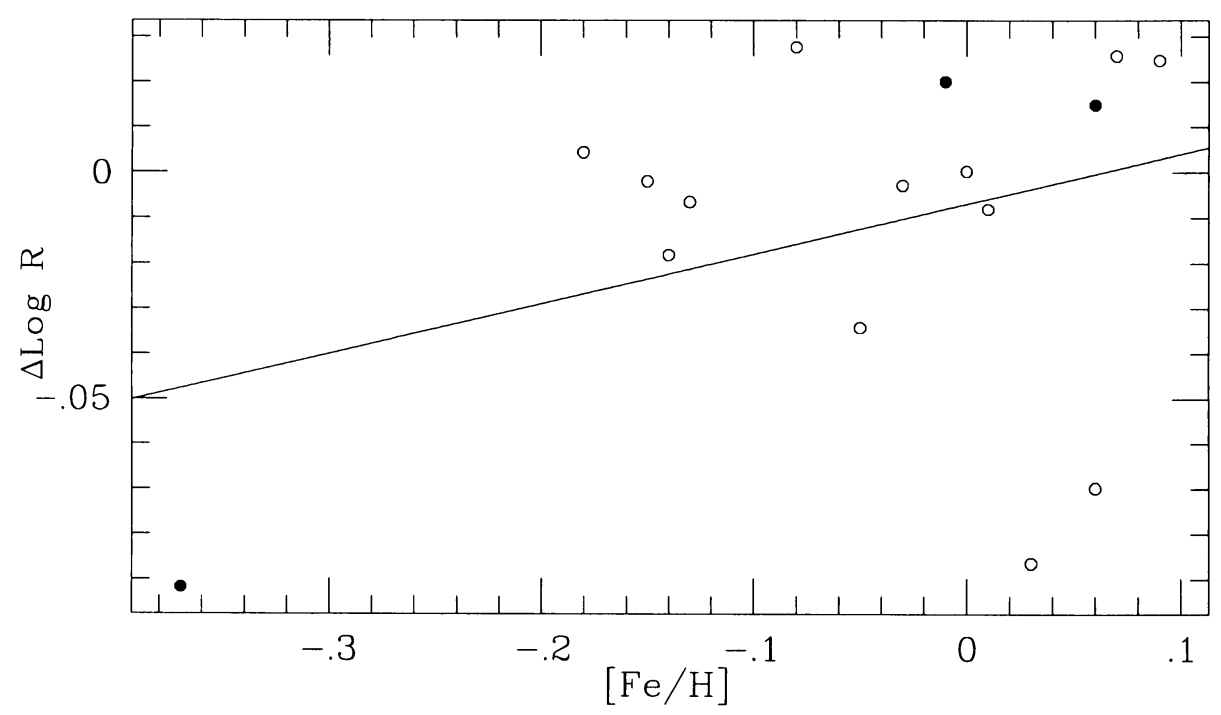

Figure 2. $\Delta R\left(\log R_{\text {predicted }}-\log R_{\text {empirical }}\right)$ vs. [Fe/H]. $\Delta R$ values were calculated using the period-radius relation in Fig. 1 , and the three overtone Cepheids (filled circles) have been shifted into zero point agreement with the fundamental pulsators (open circles). The point at lower left represents EV Sct; those at lower right TW Nor and SV Vul.

But could low-metallicity Cepheids be cooler? This seems unlikely in view of the results cited in Laney \& Stobie (1994), who found that the temperature shift between Galactic and SMC Cepheids was equivalent to a shift in $B-V$ at a given period of $0.081 \pm 0.016$ (SMC bluer) after the effects of line blanketing had been removed. The same shift appears in every waveband, and for $V-I_{C}$ (important for HST moduli), SMC Cepheids are bluer than LMC Cepheids at the same period by $0.052 \pm 0.018$ (67 stars), and bluer than Galactic Cepheids by $0.067 \pm 0.014$ (86 stars). The dereddened $V-I_{C}$ colours of Cepheids with Fry \& Carney metallicities show the same trend - the low-metallicity objects tend to be bluer at a given period (Fig. 3). (Though in this small sample the slope is not statistically significant, there is certainly no sign of a large trend toward redder colours at low $[\mathrm{Fe} / \mathrm{H}]$.)

These comparisons assume the $B V I_{C}$ reddening scale as described by Caldwell \& Coulson (1985), but no reasonable reddening values for the Cepheids involved will make the most metal-deficient population (SMC) redder than Galactic Cepheids at the same period. The empirical differences in intrinsic colour cited above are both quantitatively and qualitatively what might be expected, given the crucial equations in Iben \& Renzini (1984). On the other hand, the bluer $V-I_{C}$ colours for SMC Cepheids at a given period do clearly imply the need for a modest metallicity correction in moduli determined using a single colour to determine reddening, as argued by Sasselov et al. (1997). With 'true' reddening values and an invariant bolometric PL relation, the necessary adjustments to the $V$ modulus (Laney \& Stobie 1994) are much smaller. 


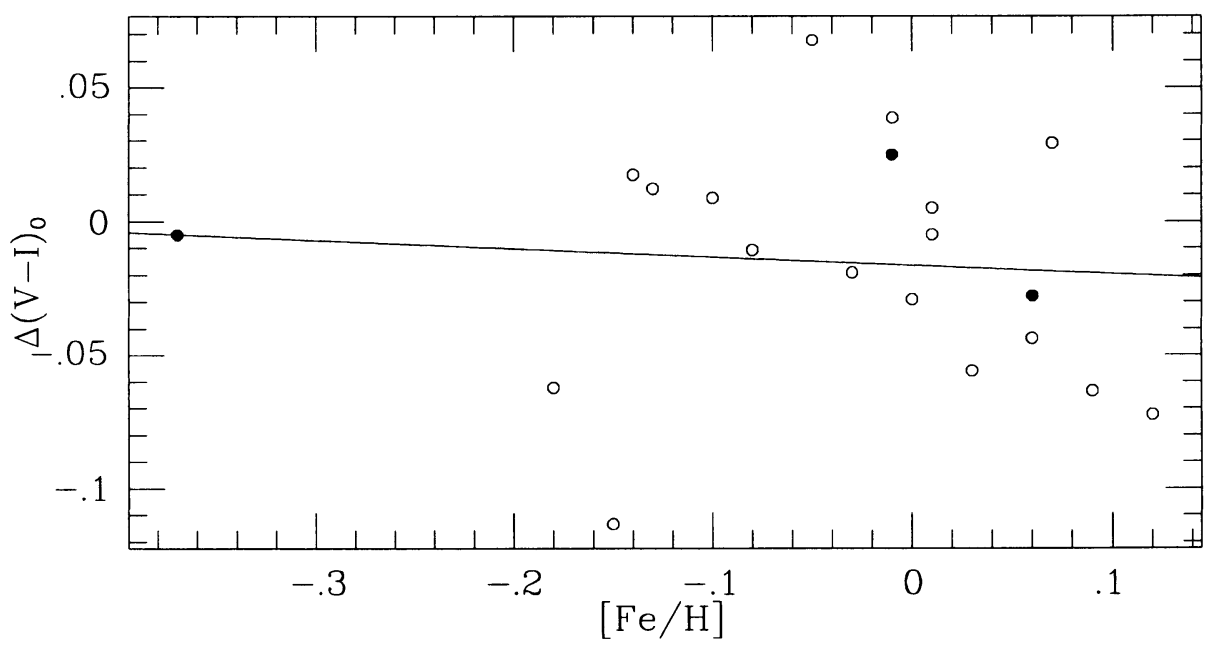

Figure 3. $\Delta\left(V-I_{C}\right)_{0}\left(\left(V-I_{C}\right)_{0(\text { predicted })}-\left(V-I_{C}\right)_{0(\text { empirical })}\right)$ vs. $[\mathrm{Fe} / \mathrm{H}]$ for all stars with Fry \& Carney (1997) metallicities and $J H K$ photometry. The $\Delta\left(V-I_{C}\right)$ values were calculated using a $\left(V-I_{C}\right)$ period-colour relation derived from 110 Cepheids, and the reddening for each star shown has been individually corrected for metallicity. Lowmetallicity Cepheids tend to be slightly bluer, as might be expected.

Combining the temperature shift results with the period-radius comparisons above makes it clear that the suggestion by Sekiguchi \& Fukugita (1998) - that LMC Cepheids may be fainter than Galactic Cepheids at the same period by $0.4 \mathrm{mag}$ - is inconsistent with the data by at least $6 \sigma$.

\section{References}

Caldwell, J.A.R., Coulson, I.M. 1985, MNRAS, 212, 879

Feast, M.W. 1991, in: Observational Tests of Cosmological Inflation, (eds.) Shanks et al., NATO ASI Series C, vol. 348, Kluwer, p. 147

Freedman, W.L., Madore, B.F. 1990, ApJ, 365, 186

Fry, A.M., Carney, B.W. 1997, AJ, 113, 1073

Iben, I., Renzini, A. 1984, Phys. Reports, 105, 329

Laney, C.D., Stobie, R.S. 1994, MNRAS, 266, 441

Laney, C.D., Stobie, R.S. 1995a, MNRAS, 274, 337 (LS95a)

Laney, C.D., Stobie, R.S. 1995b, in: Astrophysical Applications of Stellar Pulsation, (eds.) R.S. Stobie \& P.A. Whitelock, ASP Conf. Ser., 83, p. 254 (LS95b)

Sekiguchi, M., Fukugita, M. 1998, Observatory, 118, 73

Sasselov, D.D., et al. 1997, A\&A, 324, 471 


\section{Discussion}

Feast: Would not $(J-K)$ be a better metal independent index of temperature than $(V-I)_{C}$ ?

Laney: Both $(J-K)$ and $(V-I)_{C}$ are nearly invariant with metallicity at a given temperature, but $(V-I)_{C}$ colours are available for many more stars, making it possible to define the colour differences between Galactic, LMC and SMC Cepheids more accurately. 